\title{
Double Jeopardy : Acute Myocardial Infarction Complicated by Cardiogenic Shock and Contrast Mediated Anaphylactoid Reaction
}

\author{
Ha-uyenThi Nguyen PharmD, Andrew B. Civitello MD, Cihan Cevik MD, Leo Simpson MD
}

\begin{abstract}
We present the case of a 63-year-old woman who developed a severe anaphylactoid reaction to iodinated contrast during an emergency percutaneous intervention (PCI) for a large anterior wall ST elevation myocardial infarction (STEMI). She developed cardiogenic shock followed by cardiopulmonary arrest and was placed on arterio-venous extra corporeal membrane oxygenation (ECMO). While anaphylactoid/ anaphylactic reactions to radiocontrast agents have been well documented in the literature, the development of an anaphylactoid reaction secondary to radiocontrast media in a STEMI resulting in cardiogenic shock has never been reported. We discuss nonimmunologic mechanisms for anaphylactoid reactions to contrast media and the use of premedication to prevent these reactions. Studies have shown that premedication preventscutaneous reactions to iodinated contrast media (ICM), and, given the excellent safety profiles of these premedications, physicians should consider using them in patients at risk for ICM reactions.
\end{abstract}

Key words: Anaphylactoid reaction, anaphylaxis, iodinated contrast media, cardiogenic shock, acute myocardial infarction, extra corporeal membrane oxygenation

\section{INTRODUCTION}

Anaphylactoid/anaphylactic reactions to radiocontrast agents have been well documented in the literature. The incidence of severe reactions, such as shock to iodinated contrast media (ICM), is extremely low (less than one death per 100,000 patients). ${ }^{1}$ The development of an anaphylactoid reaction secondary to radiocontrast media during a ST elevation myocardial infarction (STEMI) resulting in cardiogenic shock has never been reported. We present the case of a

Corresponding author: Ha-uyen Thi Nguyen Pharm.D. Contact Information: hauyenthi@yahoo.com

DOI: 10.12746/swrccc2013.0102.021 63-year-old woman who developed a severe anaphylactoid reaction to iodinated contrast during an emergency percutaneous coronary intervention (PCl) for a large anterior wall STEMI resulting in cardiogenic shock followed by a cardiopulmonary arrest.

\section{Case Presenttion}

A 63-year-old woman with a history of obesity, hypertension, and smoking was transferred from an outside community hospital to our hospital with a large acute anterior STEMI. She presented with severe crushing precordial chest pain that began two hours prior to admission along with nausea, vomiting, and diaphoresis. She continued to have chest pain (8/10) with ECG changes consistent with a large anterior myocardial infarction and elevated cardiac en- 
zymes. The patient reported a possible allergic history to iodine. The risk of an anaphylactoid contrast reaction was explained to her, and she elected to proceed with the procedure. She was premedicated with hydrocortisone sodium succinate $100 \mathrm{mg}$ IV push and diphenhydramine $50 \mathrm{mg}$ IV push.

Her coronary angiogram demonstrated total occlusion of the mid left anterior descending (LAD) and right coronary artery (Figure 1 ) and a $70 \%$ stenosis of the left circumflex (LCX). Since the culprit lesion was in the $L A D$, it was decided to intervene in the LAD.

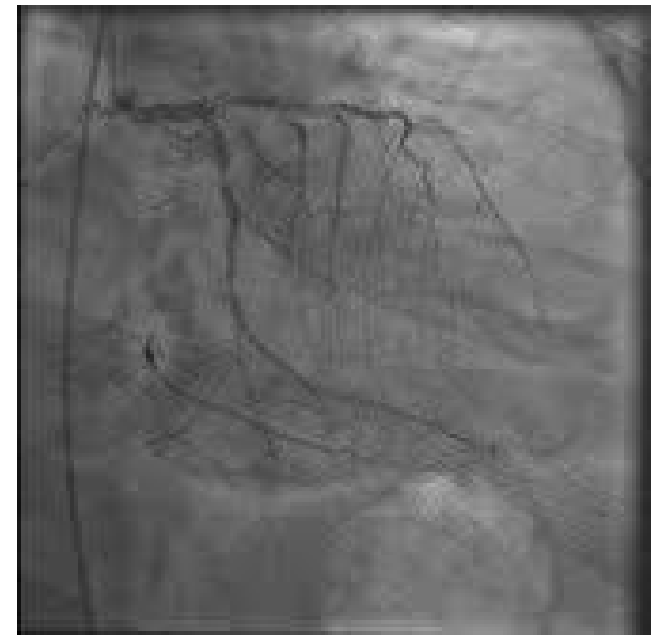

Figure $1.100 \%$ occlusion of the left anterior descending artery and right coronary artery.

Five minutes after administration of iopamidol, a nonionic low osmolality monomeric contrast agent, she complained of difficulty breathing, difficulty swallowing, hoarseness, and swelling in her throat. She also began to have extreme swelling of her upper body, neck and face. Her heart rate dropped to 50 bpm, her blood pressure dropped to $70 / 50 \mathrm{mmHg}$, and she had audible wheezing and stridor. She was intubated in the cardiac catheterization lab but rapidly deteriorated into a cardiopulmonary arrest requiring chest compressions and multiple inotropes. She progressed into pulseless electrical activity along with incessant episodes of ventricular tachycardia that were treated with electrical and pharmacologic cardioversion, including amiodarone and lidocaine.

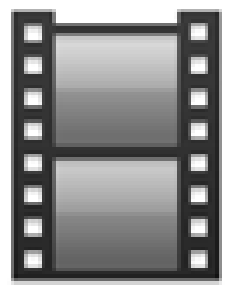

Figure 2. Movie 1 - Echocardiogram with an ejection fraction of $25-29 \%$

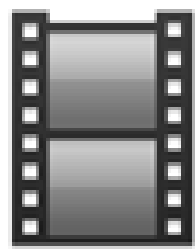

Figure 3, Movie 2 - Echocardiogram with an ejection fraction of $45-49 \%$

A Tandem Heart circuit placement was attempted but failed due to the inability to obtain transeptal access because of ongoing chest compressions. The patient was placed on arterio-venous extracorporeal membrane oxygenation (ECMO). Her early postoperative course included refractory cardiogenic shock requiring the use of multiple vasopressors,emergency aortocoronary bypass (ACB) surgery with grafts to the LAD and LCx, and acute renal failure requiring temporarydialysis. The patient was decannulated off 
ECMO after five days and needed a tracheostomy on the tenth post operative day. Her left ventricular ejection fraction improved from $25-29 \%$ (Figure 2) to 45 $49 \%$ post-ACB(Figure 3 ); she was finally discharged after a 6 week hospital course.

\section{Discussion}

The number of diagnostic cardiac catheterization and revascularization procedures performed in the United States increases each year. The American Heart Association Update on Heart Disease and Stroke Statistics reported nearly 1.3 million percutaneous interventions in 2005 alone. Integral to these procedures is the use of iodinated contrast agents that allows for proper visualization of normal and diseased cardiovascular anatomy. With the increasing use of contrast agents, it is imperative that clinicians understand and properly address the risk for and treatment of adverse reactions related to contrast use.
Anaphylaxis is an extremely rare but potentially lethal complication of cardiac catheterization andis defined as an IgE antibody mediated mast cell degranulation characterized by generalized urticaria, acute bronchospasm, and profound hypotension. Anaphylactoid reactions have indistinguishable characteristics from IgE mediated hypersensitivity, but the lack of a specific IgE to contrast media has led to the term "anaphylactoid" to describe these reactions. ${ }^{2}$ Thus, a patient can develop an anaphylactoid reaction upon the first exposure to an offending agent, unlike anaphylaxis in which the reaction can occur only after a primary exposure. In severe anaphylaxis the cardiovascular system is frequently involved with symptoms, such as hypotension, cardiovascular collapse, arrhythmias, and/ or chest pain ${ }^{3}$, making it almost indistinguishable from cardiogenic shock. Our patient developed cardiogenic shock secondary to an anaphylactoid reaction to iopamidol and to an acute myocardial infarction.

Table 1. Pretreatment protocol for contrast allergy recommended by American College of Radiology

a. Prednisone $50 \mathrm{mg}$ orally at 13 hours, 7 hours, and 1 hour before proce dure

b. Diphenhydramine $25-50 \mathrm{mg}$ intravenously, intramuscularly or by mouth 1 hour before the procedure

c. Nonionic, low-osmolality contrast medium

Table 2. Another option for suspected severe contrast mediated reaction

a. Prednisone $50 \mathrm{mg}$ orally 13,7 and 1 hour(s) prior to procedure or hydrocortisone $100 \mathrm{mg}$ intravenously 1 hour prior to procedure

b. Cimetidine $300 \mathrm{mg}$ orally 1 hour prior

c. Diphenhydramine $50 \mathrm{mg}$ orally 1 hour prior

d. Montelukast $10 \mathrm{mg}$ orally 1 hour prior

e. Nonionic low or iso-osmolar contrast agent 
The current paradigm maintains that immediate type hypersensitivity reactions to ICM are mediated by non-immunologic (i.e. non lgE) mechanisms, but the pathophysiology remains to be established. Several studies have proposed the following mechanisms of action: direct mast cell activation, high osmolality irritant-like action, and activation of the coagulation or complement cascade. In contrast, a study that used skin tests and basophil activation tests, an IgEmediated contrast material allergy was identified in four of 96 examined patients. Genuine IgE-mediated allergic anaphylaxis to contrast media is rare but can occur. In summary, an anaphylactoid reaction to contrast media does not require IgE specific antibodies to contrast media to induce a reaction, and this has led to the description of anaphylactoid reactions as quasi- or pseudoanaphylaxis.

Goss and coworkers reported that the incidence of contrast related complications in the cardiac catheterization laboratory was $0.23 \%$, with one death per 55,000 cases. $^{3}$ There is some debate surrounding whether or not we can properly identify patients at risk for an ICM reaction. This debate also extends to whether or not premedication actually prevents anaphylaxis/anaphylactoid reactions to iodinated contrast media. ${ }^{6,7}$ Studies have shown that premedication substantially reduces minor cutaneous reactions to ICM, but several prospective studies have not shown any benefit in the use of premedication for severe anaphylaxis/anaphylactoid reactions. ${ }^{6}$ In fact, although a prior reaction remains the best predictor of a future adverse event, the likelihood of a recurrent reaction is only in the range of $17 \%-35 \%{ }^{8}$. The advent of lower osmolality iodinated contrast media in the mid-1980s has contributed to the decline in anaphylaxis/anaphylactoid reactions to ICM. The consensus at this time is that a past history of an ICM reaction or a history of atopy/asthma serves as a predictor for future ICM reactions, with the former being a stronger predictor. The American College of Radiology recommends the following as a pretreatment protocol for contrast allergy(Tables 1 and 2 ). ${ }^{9}$ For emergency cases where prednisone cannot be given prior to the procedure, hydrocortisone sodium succinate $100 \mathrm{mg}$ (Solu-Cortef $($ ) should be administered at the time of the procedure.
Another component in our patient's case was her development of cardiogenic shock secondary to both the large anterior wall infarct and anaphylactoid shock. Cardiogenic shock is the most common cause of death in patients with acute myocardial infarction (AMI)with a frequency of around 7-10\%.,11,12 Severe forms of anaphylaxis affects the cardiovascular system, and clinical manifestations may appear similar to cardiogenic shock. Although there have been reports on anaphylactoid contrast reactions in the catheterization lab or cardiogenic shock following an acute myocardial infarction, we believe that ours is the first reported case of cardiogenic shock following an anaphylactoid iodinated contrast mediated reaction in the setting of a STEMI. ECMO had a significant role in the management of this patient by providing both cardiac and respiratory support during the acute course.

In conclusion, although anaphylactoid contrast reactions leading to cardiogenic shock are rare, prompt recognition and treatment is essential to prevent death. Anaphylactoid reactions, unlike anaphylaxis reactions, are not true immune mediated reactions and thus do not utilize an IgE pathway. However, anaphylactoid reactions are indistinguishable from anaphylaxis reactions. While the pathophysiology of anaphylactoid reactions remains to be fully explained, proposed mechanisms include an irritant-like effect on mediator cells. Whether or not premedication can prevent a life threatening ICM reaction is uncertain, but the safety profiles of the premedications should encourage physicians to use them. Measures should be taken to premedicate appropriate patients and monitor for any adverse event. Clinicians should keep in mind that premedication does not completely prevent ICM reactions. In addition, anaphylactoid reactions do not require specific antibodies to contrast media to occur and thus can occur upon initial exposure. In our patient's case, appropriate measures were taken yet an anaphylactoid contrast mediated reaction leading to cardiogenic shock occurred. Cardiac and respiratory support with A-V ECMO seemed useful in this case and might improve outcomes in such critically ill patients. 
Author Affiliation: Ha-uyen Thi Nguyen PharmD, Baylor College of Medicine; Andrew B. Civitello MD, Interventional Cardiology, Saint Luke's Episcopal Hospital; Cihan Cevik MD, Interventional Cardiology, Saint Luke's Episcopal Hospital; Leo Simpson MD, Interventional Cardiology, Saint Luke's Episcopal Hospital.

Received: 02/13/2013

Accepted: 03/06/2013

Reviewers: Kenneth Nugent MD, Jason Wischemeyer MD

Published electronically: 04/15/2013

Conflict of Interest Disclosures: NONE
10. Khalid L, Dhakam SH. A review of cardiogenic shock in acute myocardial infarction. Curr Cardio Reviews 2008; 4: $34-40$.

11. Goldberg RJ, Gore JM, Alpert JS, et al. Cardiogenic shock after acute myocardial infarction. Incidence and mortality from a community-wide perspective, 1975-1988. N Engl J Med 1991; 325: 1117-22.

12. Braunwald EB. Hemodynamic disturbances in acute myocardial infarction. In: Brainwald EB, editor. Heart disease. Philadelphia: W. B. Saunders 1997; 1233-45.

\section{REFERENCES}

1. Sakakibara M, Tsutsui H. Successful non contrast percutaneous coronary intervention for patient with unstable angina and prior anaphylactic reaction to iodinated contrast medium. Inter Med 2009; 48: 1753-1757.

2. Nayak KR, White AA, Cavendish JJ, Barker CM, Kandzari DE. Anaphylactoid reactions to radiocontrast agents: prevention and treatment in the cardiac catheterization laboratory. J Invasive Cardiol 2009; 21: 548-551.

3. Goss JE, Chambers CE, Heupler FA Jr. Systemic anaphylactoid reactions to iodinated contrast media during cardiac catheterization procedures: Guidelines for prevention, diagnosis, and treatment. Laboratory Performance Standards Committee of the Society for Cardiac Angiography and Interventions. Cathet Cardiovasc Diagn 1995; 34: 99-104.

4. Wasserman NH. Anaphylactoid reactions to radiopaque contrast dye. Mayo Clin Proc 2009; 84 (7):663-664.

5. Trcka J, Schmidt C, Seitz CS, et al. Anaphylaxis to iodinated contrast material: nonallergic hypersensitivity or IgE-mediated allergy? Am J Radiol 2008; 190: 666-670.

6. Bettmann MA, Heeren T, Greenfield A, Goudey C. Adverse events with radiographic contrast agents: results of the SCVIR Contrast Agent Registry. Radiology 1997; 203: 611-620.

7. Bettmann MA. Frequently asked questions: iodinated contrast agents. Radiographics 2004; 24: S3-S10.

8. Bush WH, Swanson DP. Acute reactions to intravascular contrast media: Types, risk factors, recognition, and specific treatment. Am J Roent 1991; 157: 1153-1161.

9. American College of Radiology Committee on Drugs and Contrast Media. ACR Manual on Contrast Media, 5th ed. Reston, VA: American College of Radiology 2004; 5-77. 